\title{
Obscured AGN
}

\author{
Amy Barger \\ University of Wisconsin-Madison, USA \\ Email: barger@astro.wisc.edu
}

\begin{abstract}
Obscured AGN may correspond to a substantial fraction of the supermassive black hole growth rate. I will present new surveys with the SCUBA-2 instrument on the James Clerk Maxwell Telescope of the Chandra Deep Fields and discuss whether we can distinguish obscured AGN in hard X-ray and radio selected samples using submillimeter observations.
\end{abstract}

